\title{
Role of Antiviral Prophylaxis and Infection Control Measures in Elimination of Influenza -A- Nosocomial Infection among Unvaccinated Patients and Healthcare Workers
}

\author{
${ }^{1,2}$ Wafaa S. Hamza, ${ }^{3,4}$ Samar S. Morsi, ${ }^{5}$ Ebtehal S. Al Roomi \\ ${ }^{1}$ Department of Public Health and Community Medicine, Faculty of Medicine, Assiut University, \\ Egypt, ${ }^{2}$ Infection Control Office, Chest Diseases Hospital,Kuwait, ${ }^{3}$ Department of Microbiology \\ and Immunology, Faculty of Medicine, Zagazig University, Egypt, ${ }^{4}$ Infection Control Offices, Al- \\ Razi Hospital, Kuwait, ${ }^{5}$ Department of Microbiology, Chest Diseases Hospital, Kuwait.
}

Received: February, 2018 Accepted: May. 2018

\begin{abstract}
:
Background: Influenza is primarily a community-based infection; it poses threats inside healthcare facilities and can cause outbreaks. Healthcare workers (HCWs) are at risk of acquiring influenza as they act as source of infection for patients. Preventing the spread of influenza in healthcare settings is an important element to improve patient safety. Method: A prospective cohort study was conducted to analyze the nosocomial transmission of influenza A (H1N1) virus infection in 25 beds in cardiology ward in a tertiary care hospital. Results: The epidemiological investigation identified 7 cases (4 male patients and 3 female HCWs) all developed new onset of fever and cough. While $50 \%$ of them had throat irritation and none of them had gastrointestinal manifestations. All cases were confirmed as H1N1 viral infection using real-time reverse transcription PCR from 6-12 April 2015. Cases age range was 48-54 years old for patients and 32-36 years old for HCWs. All patients have ischemic heart disease and HCWs were all healthy. None of them were vaccinated, all cases received oral Oseltamivir and got favorable outcome. Conclusion: Antiviral prophylaxis and treatment (when indicated) play essential roles in controlling transmission of healthcare associated influenza and implementing rigorous infection control practices minimize cross-transmission and successively control influenza outbreak.
\end{abstract}

Key Words: Nosocomial Influenza, H1N1, Antiviral prophylaxis, epidemiological investigation, Infection control measures.

Corresponding Author: Dr. Wafaa Seddik Hamza

E-mail: wafaa95@gmail.com

\section{Introduction}

There are three types of influenza viruses: types A, B, and C. only type -A- influenza viruses cause pandemics. Seasonal influenza outbreaks can be caused by either type A or type B influenza viruses. Influenza type $C$ viruses cause mild illness in humans. ${ }^{1}$ Only type -A- is divided into subtype, based on the presence of two surface proteins: hemagglutinin $(\mathrm{H})$ and neuraminidase $(\mathrm{N})$. There are different hem- agglutinins and neuraminidase surface proteins have been identified in influenza -A- viruses (e.g. H1N1). Influenza -A- viruses vary in virulence, 
infectivity to specific hosts, modes of transmission, and the clinical presentation of infection. ${ }^{1}$ Influenza is primarily a community-based infection that is transmitted in households and community settings. $^{2}$ Influenza poses special hazards inside healthcare facilities and can cause outbreaks. Healthcare workers (HCWs) are at risk of acquiring influenza and thus serve as an important reservoir for cared patients. ${ }^{3}$ Outbreaks of influenza A have been reported in general wards, pediatric units and in neonatal intensive care units. ${ }^{4}$ Traditionally, influenza viruses have been thought to spread from person to person primarily through large-particle respiratory droplet transmission. Transmission via large-particle droplets requires close contact between source and recipient persons, (approximately 6 feet or less) through the air. Indirect contact transmission via $\mathrm{HCW}$ hands from contaminated surfaces or objects to mucosal surfaces of the face may also occur. Airborne transmission through small particle aerosols in the area of the infectious individual may also occur. ${ }^{2}$

Preventing the spread of seasonal influenza in healthcare settings is an important element of any effort to improve patient safety. While influenza infections in patients and HCWs often go undiagnosed or under-reported. ${ }^{5}$ Contact between patients and HCWs at healthcare facilities increase their risk for exposure to and transmission of preventable respiratory infections. Nosocomial transmission of respiratory infections has been reported in both acute and long-term healthcare settings. Transmission has occurred from patients to HCWs, from HCWs to patients, and among HCWs. ${ }^{5}$

Vaccination of both patients and their contacts is the cornerstone of efforts to prevent influenza transmission. ${ }^{6}$ WHO remains to recommend antiviral treatment with neuraminidase inhibitors for influenza, for patients with suspected or confirmed H5N1 or H7N9 virus infection; antiviral treatment should not be hindered while laboratory test results are waiting. The same recommendation applies to infection with influenza $A(\mathrm{H} 1 \mathrm{~N} 1)$ as it can cause severe complications. ${ }^{7}$

Post exposure prophylaxis is a successful strategy when commenced in the first 48 hours after exposure to a contact with suspected or laboratory confirmed influenza. Contacts are considered infectious beginning from 24 hours before illness onset until the end of fever. ${ }^{7}$ Chemoprophylaxis in outbreak control combined with antiviral treatment of sick people in addition to other infection control measures are recommended for controlling outbreaks of influenza. ${ }^{8}$

Objective: To analyze the nosocomial transmission of influenza-A- $(\mathrm{H} 1 \mathrm{~N} 1)$ virus infection in a tertiary care hospital and to describe the role of antiviral prophylaxis and infection control measures to control this outbreak.

\section{Methods}

Design: A Prospective cohort study with ongoing daily epidemiological and microbiological surveillance for influenza among patients and HCWs.

Setting: A 25 bed adult male cardiology ward receives care by 36 nurses and 7 doctors. The ward has 17 rooms each one has hand operated hand washing station, 8 of the rooms are double occupancy and 8 are private rooms in addition to an airborne isolation room. It is located in Chest Diseases Hospital that is 358 beds tertiary care hospital and is specialized in management of cardiac and cardiothoracic patients in the state of Kuwait.

Study population: There were two study populations 1. Twenty five adult (more than 18 years old) male patients admitted to the study hospital with different cardiac diseases and stayed in the cardiology ward to receive medical management; the majority of them were admitted in the 
hospital either before or after cardiac catheterization procedure in the form of diagnostic or therapeutic coronay angiography followed by insertion of cardiac stents. 2 . Forty three adult healthy healthcare workers (7doctors and 36 nurses) providing the medical care for their patients twenty four hours in the form of 3 shifts a day. The nurses' patient ratio was one/two or three according to the patients' medical condition. Doctors do daily round in the ward and examine all patients.

Sample size: Investigation of the outbreak among all patients residing in the cardiology ward and all healthcare workers providing care for them during the outbreak period.

Data Collection tools: Three forms were used to collect data. Surveillance form that included patient name, age, gender, nationality, hospital file number, ward, room and bed number. Date of admission, date of cardiac procedure, type and date of insertion and removal of invasive devices. Diagnosis on admission, history in details, underlying condition, clinical, laboratory and radiological evidence. Type of laboratory samples, collection date and report result. The used medications name and dosage, daily progress of the condition and patient Outcome. Outbreak notification form that included the following information: type of outbreak, Incubation period, etiological agent, mode of transmission, date of outbreak was detected /reported to infection control, outbreak location (ward). Health care facility source, Index case identified or not, if healthcare facility source was the index case; if it was a HCW or not. Date of onset of the first ill person, date of outbreak commenced, total number of affected cases; patients or staff, number of laboratory confirmed cases, number of patients still hospitalized, number of deceased patients. Outbreak status; ongoing or controlled, date of onset of last till person resolved, the date of outbreak completed. Outbreak case list: this form is the summary information of the previous two forms.

Data collection: Infection control team collected all cases' information from medical files, laboratory reports and nursing notes using the previous forms through their daily visits to the study location. Data for the current research was retrieved from the surveillance form, outbreak notification form and outbreak case list for the diagnosed nosocomial influenza cases.

Laboratory work- up: $\mathrm{H}_{1} \mathrm{~N}_{1}$ virus was identified in the nasal and throat swabs of all cases by using real-time reverse transcription PCR. The test swabs were forwarded per hospital protocol to the central reference laboratory (Virology laboratory) for polymerase-chain reaction (PCR). ${ }^{9}$ cDNA production and specific amplification of each target gene was conducted in one tube, using the Superscript III RT/Platinum Taq mix (Invitrogen, Carlsbad, CA, USA) and all reactions were executed using a Light Cycler 2.0 (Roche). Influenza testing was performed using the three-target detection system (universal influenza $A$ via the matrix gene, seasonal $\mathrm{H} 1$ and seasonal $\mathrm{H} 3$ ) developed by CDC, Atlanta [10]. The human RNase $\mathrm{P}$ gene was used as a positive control. For all rt-RT-PCR assays a negative (no template) control and positive template control were included for each primer set as per the established protocols.

Case definition: A confirmed case of swine influenza A (H1N1) virus infection is defined as a person with an acute febrile respiratory illness with laboratory confirmed swine influenza -A (H1N1) virus infection at approved laboratories by one or more of the following tests: Real Time PCR, viral culture, four-fold rise in swine influenza A (H1N1) virus specific neutralizing antibodies [11]. 
Outbreak control measures: conduct preliminary investigations, confirm the diagnosis, establish the existence of an outbreak, call for outbreak control meeting, define and identify cases, describe the data in terms of time, place, and person and implementing the needed control measures.

Investigation of contacts and follow up: The identified cases as well as all defined close contacts, HCWs in this ward, patients sharing the same ward; all were placed under daily active surveillance from the beginning of diagnosis of the first case and continue for one week after the date of diagnosis of the last case.

A total of 57 close contacts were recognized; including 2 of the patients in the same ward, 43 HCWs, 2 physiotherapists and 10 household members. All close contacts received oseltamivir chemoprophylaxis. And both groups (patients and HCWs) were monitored for development of symptoms. Any contact develops suggesting signs and symptoms were tested for Influenza A. All throat/ nasal swab samples collected during the outbreak period from these contacts revealed negative result for influenza -A- viruses by PCR. No household members were known to be affected. For all HCWs and the remaining patients, strict droplet precautions were implemented, and the individuals were required to wear surgical masks at all times. Hand hygiene ( $\mathrm{HH})$ was reinforced.

Statistical analyses: Attack rate for the outbreak was calculated by dividing the total number of influenza cases among patients by the total number of patients residing in the ward. And the attack rate among HCWs was calculated by dividing the total number of influenza cases among HCWs by the total number of HCWs providing medical care during the outbreak.

\section{Ethical consideration:}

All data were coded anonymously. Respiratory samples were collected and sent to the laboratory as part of the surveillance. The patients' identities were not disclosed at any stage and access to such data was restricted. Informed consent was obtained from all participating patients and HCWs by the investigators. Approval was taken from hospital director and infection control directorate.

\section{Results}

From 6-12 April 2015 an epidemiological investigation identified a total of 7 cases (4 male cardiac patients and 3 female HCWs), who developed new onset of symptoms that fulfilled the case definition of influenza-like illness. The clinical characteristics and timeline of the outbreak are shown in table (1) and figure (1).

H1N1 Outbreak was confirmed on 8 April 2015, after identification of three cases.

\section{Description of the outbreak}

Patients: The index case was community acquired influenza A (H1N1) virus infection confirmed in a 54 years old male patient has ischemic heart disease (IHD) admitted to the cardiology ward in 4 April with fever and cough, aerosol-generating procedures conducted. Next day nasal/ throat swabs sent for testing and it came positive on 6 April for Influenza- A(H1N1). Three other male patients acquired influenza A (H1N1) virus infection during the period from 6-11 April with age range $48-54$ years old, all of them have IHD, and 25\% (1patient) has additional cardiomyopathy. All patients presented with fever and cough while $50 \%$ of them have throat irritation and none of them has gastrointestinal manifestations. The incubation period ranged from 3-4 days after direct contact with the cases through sharing the rooms. The diagnosis was via nasal /throat swabs. All patients did not receive seasonal influenza vaccine. The calculated attack rate for patient's 
infection is $12 \%$ (3 of 25). All patients cured after receiving treatment with oral Oseltamivir $75 \mathrm{mg}$ tablets twice daily for 5 days, see table (1) and figure (1).

Healthcare workers: Three female HCWs, age range 32-36 years, developed influenza-like illness (fever, cough, chills, body ache and throat irritation) they were healthy female HCWs that were providing medical care for the first and second cases; they were diagnosed after incubation period of 2-4 days during admission of the index patient. HCWs were not vaccinated for the 2015-2016 seasonal influenza, developed influenza-like illness after established contact with the patients and continued working. Their respiratory samples for influenza testing were obtained from them and came positive for influenza A (H1N1) virus; their role could be confirmed in involvement to this apparent nosocomial spread of influenza. The calculated attack rate for $\mathrm{HCWs}$ infection is $7 \%$ (3 of 43), All HCWs have favorable outcome including one pregnant nurse. All cases received treatment in the form of oral Oseltamivir.

Transmission: The exact route of transmission from the index case patient to the second case patient remains unclear. Transmission through respiratory droplets is possible because the index case patient had cough, and he had aerosol-generating procedures conducted during his hospitalization period. Cross-infection through fomites, the unclean hands of HCWs or visitors, or contaminated medical equipment might also be possible. No personal protective equipment (PPE) was used before the second case-patients were confirmed to be positive for influenza -A- virus infection. The second patient has some psychological problems and intentionally was coughing and spitting in front of others which propagates the transmission to the rest of cases and many trails made to restrict his movements outside his room.
Actions taken to control outbreak: Immediately after declaring the outbreak a multidisciplinary outbreak team comprising infection control team, microbiology laboratory, cardiology doctors, hospital director, preventive medicine physician, medical store personnel, the assistant director of nursing and ward head nurse was established. Conducting an urgent outbreak meeting, during this meeting the situation was described and discussed in details.

Recommendations and measurers to control the outbreak were clearly settled, outlined and assigned to all the meeting personnel including the following: Notification must be done immediately for suspected or confirmed cases, Implementing strict standard as well as droplet precautions for all patients with suspected or confirmed influenza-A. Isolating suspected and /or confirmed influenza patients under droplet isolation precautions and use N95 mask during any aerosol generating procedure, Restricting patient movement and transfer to avoid spread of infection in addition to limit staff movement from areas with outbreak to other units and buildings and implement staff cohorting. Administration of influenza antiviral treatment (Oseltamivir) to all patients and administer influenza antiviral prophylaxis to people that was in direct contact with the cases. Ensuring enough supply of the required PPE especially N95 mask in case of aerosol generating procedures. Dedicate other private rooms for isolation. Instructions given to patient contacts at home to be followed up by area clinic or area hospital regarding prophylaxis and testing if developed suggesting respiratory symptoms. An urgent notification group was created of hospital director, head of laboratory, infection control team, preventive medicine, director of nursing, a member of medical store and a representative doctor of cardiology 
department to follow up the situation around the clock and implement infection control precautions immediately. Public relation employees were helping in restriction of visiting the influenza cases. Respiratory hygiene/cough etiquette includes: covering the nose/mouth with a tissue when coughing and prompt disposal of used tissue, using surgical masks on coughing patients when tolerated and appropriate, Posted visual signs in language(s) suitable to the population served with instructions to patients and accompanying family members or friends to inform staff if they have respiratory symptoms. HH after contact with respiratory secretions and spatial separation of 1 meter of persons with respiratory infections in common waiting areas when possible. An educational program for the doctors, nurses, and physiotherapists included: advocate early diagnosis, treatment, and identification of influenza focusing on adherence to standard and transmission based precautions including droplet, contact precautions and $\mathrm{HH}$ practice, the exclusion criteria for ill staff followed by regular auditing for the implementation of the influenza prevention and control measures. Active daily surveillance of infections for all patients who are at increased risk for influenza infection was performed by infection control team. Emphasis on environmental cleaning and disinfection on daily basis in addition to after patient discharge from the room, cleaning and disinfection of all environmental surfaces and disinfection of all equipment completed as per its manufacturer instructions. No more cases were detected afterwards (as shown in figure1).

\section{Discussion}

The outbreak of human infection due to the novel swine-origin influenza -A- (H1N1) virus began in Mexico in March 2009.
Because clinical symptoms of infection with the novel influenza virus do not differ from those of seasonal human influenza, there is a continued need for subtyping and laboratory confirmation. ${ }^{12}$ This report nosocomial outbreak of influenza -A(H1N1) virus infection in an adult cardiology ward, in a tertiary care hospital, it was temporally related to the use of an aerosol-generating procedure in the index patient as well as delay implementation of isolation precautions. This happened together with peculiar uncontrolled psychological behavior of the second case including but not limited to coughing and spitting in the floor.

Aerosol transmission can occur at a short range between persons but can also involve infectious agents carried for longer distances by air currents. ${ }^{13}$ Fabian and colleagues (2008) collected exhaled breath of patients with active influenza. In one third of the study subjects; exhaled breath contained influenza, and more than 87 percent of exhaled particles were $<1 \mu{ }^{14}$ Researchers reported an outbreak of seasonal influenza in an acute care setting that was attributed to aerosol transmission. An aerosol-generating device was used on the influenza index case patient. At the same time, the authors recognized difference in the indoor airflow that likely created a directional dispersion of air and possibly carried influenza aerosols to other areas of the ward. Other patients were infected following a temporal and spatial pattern of air flow initiating from the index patient as well as two of the staff. ${ }^{15}$ Decision making regarding control measures should not be delayed and the diagnosis should be based on clinical and epidemiologic characteristics until they get results of laboratory confirmation. Effective control of outbreaks in acute care facilities is challenging since several reservoirs for transmission exists, including patients, HCWs, and visitors. ${ }^{3}$ Attack rates were high when infection 
control measures were not followed ${ }^{16}$, but low when proper measures were undertaken. ${ }^{17}$

We used determined infection control measures to prevent influenza transmission in our hospital, such as administrative and source controls, and use of PPE in concordant with other research. ${ }^{18}$ The policy of isolating or cohorting patients with suspected or confirmed influenza is reinforced; submission of an aerosolgenerating procedure is permitted only in adequately ventilated single rooms, $\mathrm{HCWs}$ are instructed to wear face masks when caring for patients with respiratory infections and advised to use N-95 respirators, face shields, gloves, and gowns while performing aerosol-generating procedures and to receive annual influenza vaccines. ${ }^{18-20}$

As recommended by Siegel et al., during an outbreak, isolation of residents of closed settings should be considered for the duration of the infectious period (five days after the onset of symptoms) to limit spread to others. Cohorting of patients in separate hospital bays may be necessary. ${ }^{21}$ Public Health England, 2014 recommended that $\mathrm{HH}$ and cough etiquette are likely to be important interventions to reduce influenza spread in the community, as well as in closed settings. ${ }^{22}$ Close contact between patients, visits to a contaminated shared area, and transmission through a wandering, sick visitor were unlikely. ${ }^{15}$ In the other hand in this study psychological condition and uncontrolled movement of the second case played an important role in transmission. In the current study all nosocomial infected patients and HCWs did not receive influenza vaccine, Patrick Ayscue et al, 2014 recommended vaccination to group infection, and found that treatment with Oseltamivir reduced the severity of the infection in general population besides vulnerable groups. ${ }^{29}$ at higher risk for influenza complications, including adults aged $<65$ years with underlying medical conditions. ${ }^{23}$

Influenza outbreaks were identified in Michigan nursing homes by Monto and his colleagues; among 8 outbreaks in which mass antiviral prophylaxis was started, no further cases were seen in units using prophylaxis in 5 locations, and the outbreak was terminated after few additional cases in the other 3 locations. While, cases continued to occur for almost a month before the beginning of antiviral prophylaxis, and cases continued to occur until $40 \%$ of residents were affected in 1 facility in which prophylaxis was not started. $^{24}$

Antiviral medications with activity against influenza viruses are an important adjunct to the infection control measures in the control of influenza. In randomized placebo controlled trial, they concluded that oseltamivir was effective in the prevention of influenza illness among persons administered chemoprophylaxis after exposure to a household member or other close contact that had laboratoryconfirmed influenza. ${ }^{25}$

The present successful use of antiviral therapy in controlling influenza outbreak is in concordant with; a meta-analysis conducted by Falagas and his colleagues and concluded that antiviral drugs seemed to reduce influenza-related complications $^{26}$, randomized control trials on administration of antiviral therapy for patients with seasonal influenza A viruses shown reduction in the duration and severity of uncomplicated laboratoryconfirmed influenza ${ }^{27,28}$ in addition to the observational studies conducted in Hong Kong on patients with influenza A (H1N1)

Querci et al. reported that former prophylaxis with oseltamivir was associated with a lower risk of developing $\mathrm{H}_{1} \mathrm{~N}_{1} \cdot{ }^{30}$ Influenza may be prevented or rendered less severe by post-exposure 
prophylaxis with antivirals (oseltamivir and zanamivir). ${ }^{27}$ In agreement with our local practice when influenza is circulating, antivirals are offered to all contacts from patients and HCWs who have had proven close contact with people with confirmed or suspected influenza.

\section{Conclusion}

Antiviral prophylaxis and treatment (when indicated) play essential roles in the reduction of transmission of healthcare associated influenza and implementing rigorous infection control practices minimize cross-transmission and successively control influenza outbreak.

\section{References}

1. OSHA; Occupational Safety and Health Administration. Pandemic Influenza Preparedness and Response Guidance for Healthcare Workers and Healthcare Employers" U.S. Department of Labor OSHA, 2009; 3328-05R.

2. Center for Disease Control and Prevention. CDC Guidance for both seasonal influenza and the Interim Guidance on Infection Control Measures for $2009 \mathrm{H} 1 \mathrm{~N} 1$ Influenza in Healthcare Settings.

3. Salgado CD, Farr BM, Hall KK, Hayden FG. Influenza in the acute hospital setting. Lancet Infect Dis., 2002, 2: 145-55.

4. Enstone JE, Puja R. Myles, Peter J.M. Openshaw, Elaine M. Gadd, Wei Shen Lim, Malcolm G. Semple, et al., Nosocomial pandemic (H1N1) 2009, United Kingdom, 2009-2010. Emerg Infect Dis. 2011.

5. Voirin N, Barret B, Metzger M, Vanhems P. Hospital acquired influenza: a synthesis using the Outbreak Reports and Intervention Studies of Nosocomial Infection (ORION) statement. J Hosp Infect. 2009; 71: 1-14.

6. SHEA; Society for Healthcare Epidemiology of America. Position Paper:
Influenza Vaccination of Healthcare Personnel; Thomas R. Talbot; Hilary Babcock; Arthur L. Caplan; Deborah Cotton; Lisa L. Maragakis; Gregory A. Poland, Edward J. Septimus; Michael L. Tapper; David J. Weber. Infection Control \& Hospital Epidemiology October 2010, Vol. 31, No. 10.

7. World Health Organization WHO Guidelines for Pharmacological Management of Pandemic (H1N1) 2009 Influenza and Other Influenza Viruses. Geneva: World Health Organization, 2010. 8. Centers for Disease Control and Prevention. Interim Guidance for Correctional and Detention Facilities on Novel Influenza A (H1N1) Virus. Available

at: http://www.cdc.gov/h1n1flu/guidance/cor rectional_facilities.htm (Accessed October 11, 2013).

9. Center for Disease Control and Prevention. CDC protocol of real time RTPCR for influenza A (H1N1).Geneva: World Health Organization, April (2009): Accessed July 23, 2009, at http://www.who.int/csr/resources /publications/ swineflu/realtimeptpcr/en /index.html.

10. Selvaraju SB and Selvarangan R. Evaluation of three influenza A and B realtime reverse 2010.

11. World Health Organization WHO case definition, (2009): at http://www.who.int /csr/resources publications/swineflu/ diagnostic_recommendations/en/index.htm 1

12. Cutler J, Schleihauf E, Hatchette TF, Billard B, Watson-Creed G, Davidson $\mathrm{R}$, et al., Investigation of the first cases of human-to-human infection with the new swine-origin influenza A (H1N1) virus in Canada. CMAJ. 2009; 181:159-63.

13. Institute of Medicine (US) Committee on Personal Protective Equipment for Healthcare Personnel to Prevent Transmission of Pandemic Influenza and Other Viral Respiratory 
Infections: Current Research Issues; Larson EL, Liverman CT, editors. Preventing Transmission of Pandemic Influenza and Other Viral Respiratory Diseases: Personal Protective Equipment for Healthcare Personnel: Update 2010. Washington (DC): National Academies Press (US); 2011. Available from: https://www.ncbi.nlm.nih.gov/books.

14. Fabian, P., J. J. McDevitt, W. H. De Haan, R. O. P. Fung, B. J. Cowling, K. H. Chan, G. M. Leung, and D. K. Milton. Influenza virus in human exhaled breath: An observational study. PLoS ONE 3(7):e2691.

15. Wong, B. C., N. Lee, Y. Li, P. K. Chan, H. Qiu, Z. Luo, R. W. Lai, K. L. Ngai, D. S. Hui, K. W. Choi, and I. T. $\mathrm{Yu}$. Possible role of aerosol transmission in a hospital outbreak of influenza. Clinical Infectious Diseases 51，2010; 10:11761183.

16. Yeom JS, Lee JH, Bae IG, Oh WS, Moon CS, Park KH, et al., H1N1 influenza infection in Korean healthcare personnel. Eur J Clin Microbiol Infect Dis. 2011; 30:1201-1206.

17. Smit PM, Mulder JW, Ahdi M, Gerritsen R, Darma S, Smits PH, et al., Low attack rate of novel influenza A (H1N1) virus infection among healthcare workers: A prospective study in a setting with an elaborated containment plan. Int Arch Occup Environ Health. 2012; 85: 163-70.

18. Ng TC, Lee N, Hui SC, Lai R, Ip M. Preventing healthcare workers from acquiring influenza. Infection Control Hospital Epidemiology 2009; 30(3): 292295.

19. World Health Organization. Infection prevention and control in healthcare for confirmed or suspected cases of pandemic (H1N1) 2009 and influenza-like illnesses.http://www.who.int/csr/resources/ publications/swineflu/swineinfinfcont/en/i ndex.html. Accessed 1 April 2010.
20. Centers for Disease Control and Prevention CDC. Interim guidance on infection control measures for 2009 H1N1 influenza in healthcare settings, including protection of healthcare personnel. http://www.cdc. $\quad$.gov/h1n1flu /guidelines_infection_control.htm.

Accessed 1 April 2010.

21. Siegel J, Rhinehart E, Jackson M, Centers for Disease Control and Prevention. Guideline for isolation precautions: preventing transmission of infectious agents in healthcare settings. CDC, 2007.

22. Public Health England. Infection control precautions to minimize transmission of respiratory tract infections (RTIs) in the healthcare setting. PHE, 2014.

23. Patrick Ayscue, DVM, Erin Murray, Timothy Uyeki, Jennifer Zipprich, Kathleen Harriman, et al., InfluenzaAssociated Intensive-Care Unit Admissions and Deaths - California, September 29, 2013-January 18, 2014.Weekly February 21, 2014 / 63(07); 143-147.

24. Arnold S. Monto, Judy Rotthoff, Esther Teich, M. Louise Herlocher, Rachel Truscon, Hui-Ling Yen, Stephanie Elias, and Suzanne E. Ohmit. Detection and control of influenza outbreaks in wellvaccinated nursing home populations. Clin Infect Dis 2004; 39:459-64.

25. Welliver R, Monto AS, Carewicz O, Schatteman E, Hassman M, Hedrick $\mathrm{J}$, et.al, Effectiveness of oseltamivir in preventing influenza in household contacts: a randomized controlled trial. JAMA 2001; 285:748-54.

26. Falagas ME, Koletsi PK, Vouloumanon EK, et al. Effectiveness and safety of neuraminidase inhibitors in reducing influenza complications: A metaanalysis of randomized, controlled trials. J Antimicrob Chemother 2010; 65:1330-46. 27. Jefferson T, Jones MA, Doshi P, Del Mar CB, Hama R, Thompson 
MJ, Spencer EA, Onakpoya I , Mahtani KR, Nunan D, Howick J, Heneghan CJ. Neuraminidase inhibitors for preventing and treating influenza in healthy adults and children. Cochrane Database Syst Rev. 2014; (4) doi: 10.1002/14651858.

28. Matheson, N.J., Harnden, A.R., Per era, R., Sheikh, A., and Symmonds Abrahams, M. Neuraminidase inhibitors for preventing and treating influenza in children. Cochrane Database of Systematic Reviews 2007(1):CD002744.

DO1.10.1002/14651858.

29. Hayden FG. Influenza antivirals: Challenges and future directions. Plenary presentation. Options for the Control of
Influenza VII. Hong Kong, SAR China, 37 September 2010. Available at: www.controlinfluenza.com/webcasts/op tionsvii (Accessed October 11, 2013).

30. Marcia Querci, Martin E. Stryjewski, Fabián Herrera,Elena Temporiti,Wanda Alcalá,Natalia Chavez,Laura Figueras,Fernanda Barberis,Marcela Echavarría,Cristina Videla,Alfredo Martínez,Guadalupe Carballal \&Pablo Bonvehí Healthcare personnel infected with novel influenza A H1N1 virus in university hospitals in Buenos Aires, Argentina. Scand J Infect Dis. 2011 Jan; 43(1):70-74. 
Table (1): Description of Cases of Influenza A (H1N1) Nosocomial Infection in Male Cardiology Ward

\begin{tabular}{|c|c|c|c|c|c|c|c|c|c|c|c|}
\hline \multirow[b]{2}{*}{ No } & \multirow[b]{2}{*}{ Age } & \multirow[b]{2}{*}{ Gender } & \multirow[b]{2}{*}{ IP } & \multicolumn{2}{|c|}{ Date } & \multirow[b]{2}{*}{ Vaccine } & \multirow{2}{*}{$\begin{array}{c}\text { Underlying } \\
\text { risks }\end{array}$} & \multirow{2}{*}{$\begin{array}{l}\text { Clinical } \\
\text { picture }\end{array}$} & \multirow[b]{2}{*}{ Cases } & \multirow{2}{*}{$\begin{array}{l}\text { Anti- viral } \\
\text { medicine }\end{array}$} & \multirow[b]{2}{*}{ Outcome } \\
\hline & & & & $\begin{array}{l}\text { Onset of } \\
\text { S. and S. }\end{array}$ & $\begin{array}{c}\text { Lab } \\
\text { diagnosis }\end{array}$ & & & & & & \\
\hline 1 & 54 & $\mathrm{M}$ & index & 4 April & 6 April & No & IHD & $\begin{array}{l}\text { Fever, } \\
\text { cough }\end{array}$ & patient & yes & Recovered \\
\hline 2 & 48 & $\mathrm{M}$ & $3 d$ & 6 April & 8 April & No & $\begin{array}{l}\text { IHD, } \\
\text { Psychiatric \& } \\
\text { Cardiomyopathy }\end{array}$ & $\begin{array}{l}\text { Fever, } \\
\text { cough }\end{array}$ & patient & yes & Recovered \\
\hline 3 & 56 & $\mathrm{M}$ & $3 d$ & 7April & 8 April & No & IHD & $\begin{array}{l}\text { fever, } \\
\text { Cough, } \\
\text { throat } \\
\text { irritation }\end{array}$ & patient & yes & Recovered \\
\hline 4 & 36 & $\mathrm{~F}$ & $2 d$ & 8 April & 9 April & No & - & $\begin{array}{l}\text { Fever, } \\
\text { cough }\end{array}$ & HCW & yes & Recovered \\
\hline 5 & 32 & $\mathrm{~F}$ & $4 d$ & 8 April & 9 April & No & - & $\begin{array}{l}\text { Fever, } \\
\text { Chills, } \\
\text { throat } \\
\text { irritation }\end{array}$ & HCW & yes & Recovered \\
\hline 6 & 54 & $\mathrm{M}$ & $4 d$ & 8 April & 11 April & No & IHD & $\begin{array}{l}\text { Fever } \\
\text { cough }\end{array}$ & patient & yes & Recovered \\
\hline 7 & 35 & $\mathrm{~F}$ & $4 d$ & 11 April & 12 April & No & Pregnant & $\begin{array}{l}\text { Fever, } \\
\text { Cough, } \\
\text { body ache }\end{array}$ & HCW & yes & Recovered \\
\hline
\end{tabular}

IP: Incubation Period; IHD: Ischemic Heart Disease; S. and S.: Signs and Symptoms 


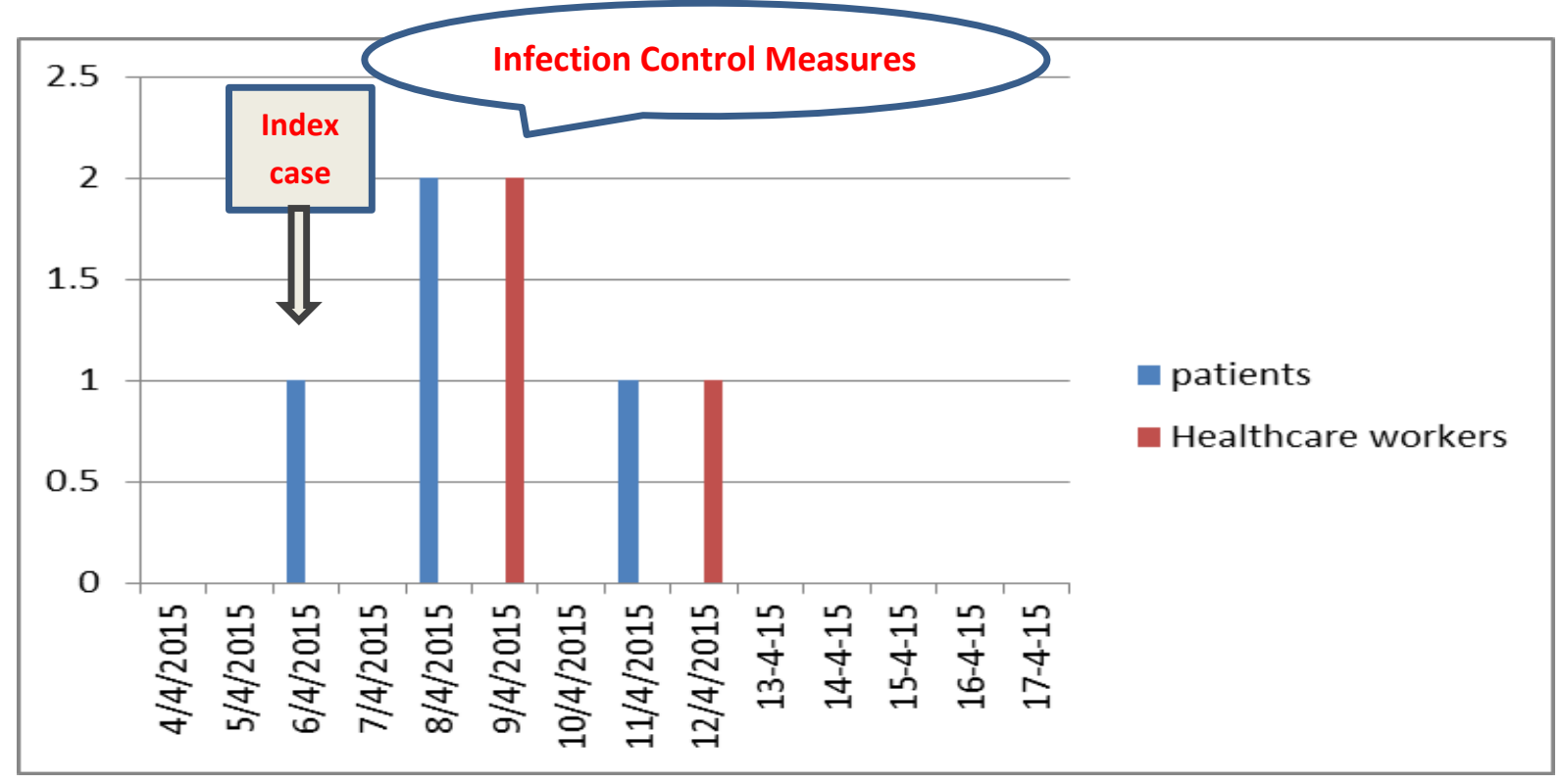

Figure (1): Epidemic Curve of Influenza -A- (H1N1) Nosocomial Infections by Date of diagnosis and Timeline 